\title{
" L'Homme à qui je devais le plus après mon père et ma mère " : Léon Bloy disciple de Jules Barbey d'Aurevilly
}

Si Bloy consacre de nombreux textes ${ }^{1}$ au Connétable des Lettres, la réciproque n'est pas vraie ${ }^{2}$. Cette asymétrie, bien qu'anecdotique, constitue un point de départ révélateur de la nature de la relation qu'entretiennent nos deux vitupérateurs du monde moderne que trente-huit ans séparent. La différence de génération est, à l'évidence, une condition favorable à la mise en place d'une relation qui, si elle peut sembler à première vue relever d'un rapport d'admiration unilatéral, prend très vite la forme d'une relation d'autorité de maître à disciple. Ce rapport architectonique est explicitement thématisé chez Bloy qui reconnait volontiers en Barbey un " maître adoré " (1965, p. 24), son « vieux maître » (2012, p. 474), ce " pauvre vieux maître » (2012, p. 563) auquel celui qui semble son disciple, et qui s'éprouve comme tel, veut rendre un hommage posthume en écrivant un livre à sa gloire. Si ce livre avait vu le jour, il aurait eu pour titre Le Pauvre Maître ${ }^{3}$.

Cette relation de plus de vingt ans, parfois conflictuelle $e^{4}$, conserve un certain flou tout en étant bien documentée : nulle peine à retracer les grandes étapes de celle-

- Yoann Chaumeil - professeur agrégé, doctorant à l'université Toulouse-Jean Jaurès sous la codirection de Fabienne Bercegol et de Pierre Glaudes. Adresse de correspondance : 20 Avenue Alain Gerbault, 31100 Toulouse, France ; e-mail : chaumeil.yoann@laposte.net

ORCID iD : https://orcid.org/0000-0003-2844-6664

1. Nous songeons au poème en prose La Méduse-Astruc, au premier chapitre d'Un Brelan d'excommuniés, ainsi qu’aux articles de critique littéraire que Bloy a écrits au sujet de l'œuvre aurevillienne.

2. Barbey a, en tout et pour tout, accordé une préface au premier livre de Bloy sur Christophe Colomb, Le Révélateur du Globe.

3. Voir la belle page que Bloy consacre à ce sujet dans son Journal, à la date du 3 décembre 1902.

4. Plusieurs événements les éloignent, parmi lesquels la relation mystique qu'entretient Bloy avec Anne-Marie Roulé de 1877 à 1882, son amitié pour Huysmans, la place que prendra Louise Read dans la vie de Barbey, le silence coupable de ce dernier au moment de la publication du Désespéré. 
ci ; plus encore, on décèle sans mal les influences déterminantes de l'un sur l'œuvre et sur la vie de l'autre. Plusieurs articles ont été écrits au sujet de leur relation, notamment au début des années $1990^{5}$. L'accent a souvent été mis sur l'influence qu'a pu avoir Barbey sur Bloy, à un niveau aussi bien philosophique, politique, que littéraire, établissant entre eux une véritable "parenté d'imagination" (Petit, 1971, p. 10). Si malgré cela l'impression de flou demeure, c'est en partie parce que nos deux écrivains eux-mêmes, lorsqu'ils sévoquent l'un l'autre, laissent une large place à l'image, comme si leur relation avait une puissance telle qu'elle ne pouvait que résister à la rationalité du discours, comme si elle devait nécessairement en passer par l'image pour s'épanouir sans être diminuée. Ce qui frappe lorsqu'on se penche sur la manière dont le disciple et le maître s'appréhendent, ce sont les figures d'analogie. Nous pensons qu'il serait bien trop réducteur de cantonner celles-ci à de simples expressions littéraires et poétiques. Elles ajoutent une densité symbolique à leur relation. Cet article propose d'examiner l'hypothèse selon laquelle la relation de maître à disciple qui se joue entre ces deux auteurs - au goût particulièrement prononcé pour la pose et les masques - peut se comprendre avant tout en termes d'image et d'ethos. Ainsi, sorti du cadre fictionnel, l'emballement de l'imagination continue d'être structurant et signifiant dans la configuration de la relation : l'image pense. Elle laisse entrevoir la construction des figures du maitre et du disciple selon une logique symbolique propre à deux auteurs dont on sait combien ils s'inscrivent au sein d'une métaphysique figurative.

\section{Le charisme d'un dandy}

À la source de l'autorité qu'a, en l'occurrence, le maître sur le disciple, se trouve ce que Weber qualifie de charisme, compris comme « l'autorité fondée sur la grâce personnelle et extraordinaire d'un individu » qui entraîne « le dévouement tout personnel des sujets à la cause d'un homme " (1959, p. 114). La rencontre de Barbey fait naître chez le jeune Bloy une admiration indépassable et durable. Toute-puissante, l'image fonde le pouvoir de celui qui allait devenir le « maître bien-aimé » (Bollery, 1947, p. 261). L'ethos de Barbey éblouit le jeune Bloy qui le croise par hasard alors qu'il descend la rue Rousselet, prêt à vendre ses Voltaire que Barbey - ironie du sort - lui conseillera plus tard de lire. C'est comme une apparition : ce qui émane de Barbey ensorcelle immédiatement Bloy. Ce dernier recourt en effet à des termes aux colorations surnaturelles pour évoquer, quelque temps plus tard, l'emprise qu'a sur lui le dandy

5. Nous renvoyons notamment à l'article de Pierre Glaudes « Bloy et Barbey d'Aurevilly » paru en 1990 dans La Revue des lettres modernes ; à l'article de Sophie Parrault « Léon Bloy admirateur de Barbey d'Aurevilly » paru dans le dernier Bulletin de la Société des études bloyennes en 1991 ; à l'article de Philippe Berthier, "Léon Bloy et Barbey d'Aurevilly », paru dans le Cahier de l'Herne consacré à Bloy. 
superbe. Ainsi parle-t-il de "l'incroyable puissance de séduction et de fascination " (Bloy, 1965, p. 23) qu'il exerce sur qui l'approche. Il va jusqu’à présenter le maître sous les traits de "la Fantaisie même » et du "porte-étendard et [du] porte-foudre de la Vérité et de la Beauté quand même » $(1965$, p. 23), dans un élan antonomastique assez généreux et enlevé pour multiplier les majuscules sublimantes qui entérinent la noblesse surnaturelle du maitre. Fait notable, l'attirance initiale du jeune écrivain pour son aîné ne passe donc pas par l'intermédiaire du livre, comme ce sera le cas pour les futurs disciples de $B \operatorname{loy}^{6}$, mais par la puissance symbolique de l'image qui se dégage du personnage que Barbey campe. Cette première excitation de l'imagination - terme qui en lui-même marque, par son suffixe, la potentialité d'action des représentations - détermine non seulement la suite de leur relation, mais encore l'existence même de cette relation : elle en est la condition sine qua non.

Dans le prolongement de cette subjugation, Bloy utilise l'image de la lutte pour qualifier les premiers temps de leur rapport marqués par la conversion du disciple par le maitre. Cette lutte se présente toutefois sur un mode paradoxal, puisque tout se passe comme s'il ne cherchait aucunement à résister : il abdique en reconnaissant l'autorité de celui qui est désormais son maître. Il s'avoue volontiers vaincu au sein d'un rapport de force qui ne semble pas avoir réellement eu lieu tant l'issue en est soudaine. La lutte en elle-même est passée sous silence pour en évoquer seulement le résultat : "Il m'a vaincu, l'incroyable ascendant dont il dispose m’a transformé » (Bollery, 1947, p. 98). L'image recèle une force de condensation pour figurer un bouleversement existentiel. C'est qu'il doit au Connétable rien de moins que son retour au catholicisme ainsi que son engouement pour La Salette ${ }^{7}$. L'image de cette confrontation a quelque chose d'un cérémonial qui s'apparente à une dialectique du maître et du disciple qui se jouerait, tout comme celle du maitre et de l'esclave, en termes de reconnaissance ; celle-ci aboutit en l'occurrence à la formation d'une domination consentie, d'une autorité désirée. L'on trouve une variation autour de cette scène de soumission volontaire lorsque Bloy développe les potentialités de l'avatar du sagittaire que Barbey s'est forgé en évoquant " M. d'Aurevilly " " transperçant d'une sagète légère le mastodonte d'orgueil » (Bollery, 1947, p. 460) sous les traits duquel il se peint. C'est en de semblables termes que le jeune écrivain figure sa conver-

6. Pierre Van der Meer vient à Bloy par la lecture de La Femme pauvre, tout comme les Maritain et Georges Rouault. Pour Léopold Levaux, c'est L'Âme de Napoléon qui est déterminant.

7. Première manifestation mariale reconnue par un mandement de l'autorité épiscopale, l'apparition de La Salette à Mélanie et à son petit frère Maximim le 19 septembre 1846 constitue aux yeux de Bloy l'événement capital du XIx ${ }^{e}$ siècle. Pour la première fois depuis la mort du Christ sur la croix, la Vierge prend la parole. C'est une Vierge éplorée qui délivre aux deux jeunes bergers un message apocalyptique. Léon Bloy consacre à cette apparition pas moins de trois ouvrages, lesquels offrent à la fois un commentaire inspiré du message de la Vierge et une méditation sur la figure de Mélanie qui suivra une trajectoire prophétique le restant de sa vie. Plus largement, l'apparition est un véritable fil rouge dans l'œuvre entière de notre auteur. Initié une première fois par l'abbé Tardif de Moisdrey, il effectuera à trois autres reprises le pèlerinage sur la montagne sainte. 
sion, puisque cette flèche le « fixa comme une chouette pieuse à la porte rayonnante de l'Église de J.-C. » (Bollery, 1947, p. 460). La reprise de l'avatar du maître permet au disciple de condenser l'idée de soudaineté et de fulgurance attachée à son expérience de conversion inénarrable par la périphrase et la métaphore qui fait jouer la figure de l'animal lourd contre celle du sagittaire triomphant par sa grâce, c'està-dire, une fois de plus, par son charisme ${ }^{8}$. L'image est d'autant plus riche qu'elle fait nécessairement écho à la scène topique de la flèche envoyée par Cupidon. Dans la scène mythologique comme dans la scène biographique, qu'il soit vassal courtois ou disciple dévoué, l'être touché par la flèche se trouve engagé dans une relation asymétrique. Comparable à l'amant qui chante les louanges de sa dame après avoir été atteint par la flèche du dieu amour, Bloy écrit un poème en prose à la gloire de Barbey en guise de première œuvre littéraire.

Le disciple est en l'occurrence d'abord celui qui en adopte la posture. "Poésie sur une autre poésie " (Bloy, 1875, p. 5), comme l'écrit Barbey dans la lettre qu'il joint au manuscrit relu du poème que Bloy lui adresse ; ekphrasis sublime inspirée de la sculpture du buste de Barbey que réalisa Zacharie Astruc, La Méduse-Astruc témoigne d'abord de la position du disciple contemplant son maître qui, sculpté, offre alors « le plus prodigieux de tous les spectacles qui se puissent contempler sur la terre" (Bloy, 1965, p. 27). Le thème de la contemplation irrigue littéralement leur relation, et ce depuis leur rencontre ${ }^{9}$. Elle fait du disciple un être de désir, mais d'un désir qui ne porte pas à la consommation. Barbey se laisse comparer à une œuvre d'art ; il se met ainsi dans une position assez paradoxale : il est à la fois réifié et magnifié, sous l'œil admiratif de Bloy. Expression de l'admiration, c'est un hommage rendu de la part de celui qui contemple par le maintien d'une certaine distance, d'une distance volontaire qui se veut une juste distance, celle-là même que le spectateur d'un tableau recherche. Barbey, quant à lui, est pleinement conscient de l'admiration qu'éprouve Bloy à son endroit : " Je n'ai qu'une seule recommandation à vous faire, lui écrit-il, - vous défier de votre admiration pour moi " (Barbey d'Aurevilly, 1988, p. 132). Néanmoins, Bloy donne un tour particulier à cette position contemplative, puisque, si la contemplation est cette disposition particulière qui consiste à laisser les choses être pour elles-mêmes, sans prétendre agir sur celles-ci, lui, loin d’être passif, débride son imaginaire et fait de Barbey une figure idéalisée.

\section{La mythification réciproque}

L'autorité du maître se construit dans la durée. Pour cerner ce sur quoi celle-ci repose, encore faut-il se rendre attentif à la manière dont le faisceau d'images qui renvoient

8. Rappelons qu'étymologiquement, charisme vient du grec kháris qui signifie grâce.

9. Selon le récit proposé par J. Bollery, Bloy, à qui Barbey demande ce qu'il lui veut, répond « Vous contempler ». Et Barbey de rétorquer : « Contemplez-moi ». 
au maître s'exprime chez le disciple. L'on est frappé par la très grande unité de sens dans les images employées qui, se voulant porteuses d'une vérité ontologique, cernent Barbey avec ce même " génie de l'aperçu » que ce dernier reconnaissait à Maistre. Bloy se considère comme le disciple de celui dont l'essence lui semble celle d'un aristocrate révolté contempteur du bourgeois. Il corrobore par là l'image que le maître souhaite renvoyer de lui-même. C'est ainsi qu'il le désigne complaisamment par la mention métaphorique de héros byroniens, qu'il s'agisse de "Lara » ou de « Mazeppa » (Bloy, 1965, p. 23). L'une des périphrases sublimantes fait du maitre le "Chevalier de Dieu dans un monde sans Dieu et sans chevalerie " (Bloy, 1965, p. 23). Elle fait écho à une seconde périphrase qualifiant Barbey de "Tertullien du XIX ${ }^{\mathrm{e}}$ siècle " (Bollery, 1947, p. 86). Bloy retient donc d'abord la saveur délicieusement intempestive qu'il reconnaît au maître, encore qualifié de « catholique au milieu des incroyants, monarchiste après les monarchies, ligueur sans Ligue, gentilhomme sans roi » (Bloy, 1965, p. 23). Il souligne par là son ardeur mélancolique qui ne peut que conduire à la marginalité, position délicate dans laquelle le disciple voit un titre de gloire. L'ethos marginal que celui-ci reconnait à Barbey se retrouve dans l'image qu'il emploie pour qualifier l'auteur du Prêtre marié de " grand sorcier du vers et de la littérature » (Bollery, 1949, p. 18). Le sorcier n'est pas seulement une figure éprouvée du poète alchimiste, il est aussi celle d'une marginalité anthropologique inquiétante ${ }^{10}$. Il est ce bouc-émissaire qui, ostracisé par la communauté des hommes, en fait ressortir le fond noir. Mais Bloy raisonne avant tout en christologue. La marginalité douloureuse qu'il attribue à Barbey renvoie nécessairement à celle du Christ. Le maître, martyr, est alors le reflet du Maître, c'est-à-dire du Christ, qui fait de lui un innocent sacrifié par la communauté des hommes. Corollaire de cette innocence prêtée à Barbey, Bloy fait de son aîné une figure ambivalente de l'enfance, qui, si elle marque quelquefois l'agacement de Bloy qui souligne ainsi la versatilité et la légèreté de Barbey, n'en reste pas moins louangeuse. Le chapitre que Bloy lui consacre dans Un Brelan d'excommuniés ne s'intitule-t-il pas «L'Enfant terrible "? "Ce vieil enfant » (Bloy, 1999, p. 166) qui sait se garder de tout mensonge et de toute compromission a dans son fond «la bonté d'enfant très bon " (Bloy, 1967, p. 46).

Qu'en est-il du regard que porte le maître sur le disciple ? Il a un pouvoir normatif que n'a pas celui du disciple. L'image que Barbey a de Bloy est celle d'un croisé, d'un moine guerrier. Il serait cette " gargouille de cathédrale qui vomit les eaux du ciel sur le bons et sur les méchants " (Bloy, 1999, p. 333). Il est encore une force de la nature, un « esprit de feu » qui a une "vocation » pour « la guerre " (Bloy, 1964, p. 23), comme le Connétable l'écrit dans la préface qu'il donne au Révélateur du globe. Or, cette légende de l'Apemantus shakespearien que Barbey veut reconnaître en Bloy et que ce dernier reprendra à son compte en en faisant l'un de ses doubles romanesques, se diffuse rapidement, jusque dans les salles de rédaction. Bloy s'y conforme à travers les avatars que lui-même se crée dans le champ littéraire quelques années

10. Nous renvoyons à ce sujet aux analyses de Lévi-Strauss dans son Anthropologie structurale. 
plus tard, lorsqu'il se figure en effet sous les traits du Pal, du molosse affable, ou encore de l'entrepreneur de démolition. L'écrivain Léon Bloy, en quête de sa " personnalité littéraire » (Bloy, 2012, p. 518), naît en partie du regard que porte sur lui le maître. Lorsque notre jeune écrivain ne correspond pas tout à fait à l'image que Barbey se fait de lui, ce dernier le corrige. Il raille en effet celui qu'il appelle «Saint Bloy " (Barbey d'Aurevilly, 1988, p. 211). Il lui fait le reproche d'être cet " insupportable pédant en matière de catholicisme " (Bollery, 1947, p. 202). Ces réserves viennent mâtiner d'agacement l'admiration mutuelle, à l'image de cette déclaration qui établit un parallèle entre son disciple et Turcaret, le personnage de Lesage :

Vous avez les idées d'un moine - et d'un moine très cuistre et sans charité. [...] Turcaret le parvenu, l'enrichi d'hier, est ridicule par le faste de son luxe. Il y a, en religion, des parvenus, des enrichis d'hier qui ont le faste de leur catholicisme. Il faut prendre garde de se donner les airs de ce Turcaret-là. (Barbey d'Aurevilly, 1987, p. 226-227)

Cela nous semble exemplaire de l'action normative du maître qui consiste à « redresser » le disciple, non seulement au sens où il rectifie les erreurs de ce dernier, mais aussi au sens où il le relève, pour que celui-ci retourne sur la bonne voie. Six ans après sa mort, Bloy, loin d'avoir retiré un quelconque bénéfice de ces remontrances, confie au contraire qu'il a souffert de la dérision de son maitre en la matière : « Je lui ai paru souvent ridicule et il n'avait à me donner que des plaisanteries pour tout réconfort spirituel »(Bloy, 1999, p. 166). Malgré cela, Bloy lui resta fidèle.

\section{La fidélité au maître}

Par métonymie, Barbey, qui a engagé Bloy comme secrétaire au côté de Georges Landry, l'assimile souvent à ses propres yeux. La gamme métaphorique part de simples injonctions répétées et sans relief qui invitent Bloy à faire sérieusement son travail de relecteur pour prendre parfois une force figurative plus dense. Les "deux yeux nommés Bloy " (Barbey d'Aurevilly, 1987, p. 280) devient " mon lynx attaché " (Barbey d'Aurevilly, 1987, p. 196) dans une expression dont la dimension poétique est redoublée par la postposition d'un participe passé sans complément qui, pour l'admirateur du Grand Siècle qu'est Barbey, constitue un pastiche ironiquement lyrique du «Vénus tout entière à sa proie attachée » (Racine, 1999, p. 831). Non content de recourir au bestiaire, le maître recourt encore au registre mythologique en invitant Bloy à avoir " les cent yeux d'Argus dans [ses] deux yeux " (Barbey d'Aurevilly, 1988, p. 17). La répétition de telles images, flatteuses pour le disciple, a d'abord la fonction tout à fait pragmatique de rendre désirable un travail de relecture en lui-même assez ingrat. Mais derrière l'ingratitude de la relecture, on comprend que l'image recèle une autre profondeur : à Valognes, Barbey charge Bloy d'observer ce qui se passe dans la société parisienne et de lui en rapporter la substantifique moelle. Le disciple 
devient alors un substitut du maître car il en est une partie. Mieux, une telle image abolit symboliquement la frontière entre le maître et le disciple : l'un est un prolongement de l'autre; et si, comme le veut la tradition platonicienne, les yeux sont le miroir de l'âme, il en devient alors la partie essentielle.

Cette image nous semble symbolique de la manière dont Bloy se positionnera toute sa vie adulte par rapport à son maitre. Il n'en est pas seulement l'admirateur, il en est encore le défenseur. Peu avant la mort de l'auteur de L'Ensorcelée, Bloy empêche Péladan, considéré comme nuisible, de se rendre au chevet du Connétable mourant. Juste après sa mort, il prend garde à ce qu'une croix soit dressée sur sa tombe, contre la volonté de Louise Read : affaire très sérieuse qui occupe une partie non négligeable du début de son Journal. Bien avant la mort du maître, un caricaturiste de l'époque, Cazals, représente le disciple en chien agressif tenu en laisse. Cette image satirique est une manière certes convenue de caricaturer un disciple pris en mauvaise part. Il est vrai que Bloy n'hésite pas à avoir des altercations, parfois violentes, pour défendre son maitre. Ainsi en est-il lorsqu'il rapporte à Barbey dans sa lettre du 22 février 1876 le différend qui l'oppose à Chantrel, journaliste qui avait fait une mauvaise critique du Prêtre marié. Cette défense active du maître révèle l'extrême loyauté du disciple. Bloy rapporte une parole qu'eut Barbey avant de mourir et qui engage le disciple dans un pacte de fidélité par-delà la mort : " Je compte sur vous, me disait-il, pour me faire respecter quand je serai mort». Se sentant " le seul capable de parler de lui avec compétence », il ajoute : «Barbey d'Aurevilly n'a que moi pour le défendre » (Bloy, 1999, p. 446-447).

\section{Un rapport filial}

La puissance de leur relation est telle qu'elle vient s'inscrire par excellence dans le champ métaphorique de la relation filiale. L'inflation d'un tel imaginaire peut évidemment être mise en rapport avec la fragilisation de la sphère familiale dont nos deux auteurs ont pu faire l'expérience ${ }^{11}$. Toutefois, à l'heure de la maturité, Bloy reste mesuré et redonne la préséance à ses parents biologiques. Barbey devient « de tous les êtres humains celui à qui je devais le plus après mon père et ma mère " (Bloy, 2012, p. 506). Cette même année, date de la mort du maître, il énonce, en forme de bilan, que Barbey est "l'homme à qui je dois le plus après mon père " (Bollery, 1949 , p. 319). Nous voyons ici combien la dialectique de la reconnaissance s'est muée en une gratitude posthume. Mais, malgré ces déclarations, nul doute que Barbey a joué le rôle de père de substitution. Évidemment, la dimension intergénérationnelle de la relation n'a pu que favoriser cela. Celui qui a l'étoffe d'un père spirituel s'avère

11. Pour des raisons d'ordre idéologique, chacun a en effet renié un temps sa famille biologique en se révoltant contre elle. Plus que chez Barbey, la figure du père est chez Bloy un repoussoir absolu. Elle éclate dès le seuil de son premier roman, Le Désespéré, avec l'aveu d'un parricide. 
l'opposé en tout point du père biologique de Bloy : l'aristocrate dandy monarchiste catholique incarne l'antithèse parfaite du petit bourgeois fonctionnaire républicain franc-maçon quétait Jean-Baptiste Bloy. Barbey émancipe Bloy qui, en révolte contre le modèle paternel, se jette dans les bras du maitre. Celui-ci assume alors quelques prérogatives paternelles : à la fois modèle, mentor, tuteur, il vient compenser les défaillances d'un père repoussoir.

Nulle surprise dès lors à ce que le disciple suive une logique mimétique. Fait anecdotique et révélateur, celui-ci signe une des lettres qu'il adresse à celle qui était alors sa fiancée en empruntant la signature de Barbey, une flèche empennée, cette même flèche qui parsème le manuscrit de La Méduse-Astruc envoyé au maître et relu par ce dernier, et qui lui sert à souligner les passages qu'il trouve remarquables. Mais le mimétisme déborde bien largement le cadre de l'anecdote. Bloy forge son propre ethos, inactuel et intempestif, sur le modèle du positionnement littéraire déjà éprouvé par le Connétable. Barbey lui donne des conseils de placement dans le champ littéraire, l'invitant à ne pas imiter ses propres excès. Or, la puissance du lien entre disciple et maître se mesure ici au fait qu'en dépit des avertissements raisonnés, la logique mimétique a été plus forte. À travers l'expérience d'une marginalité assumée, Bloy trouve sa propre identité d'écrivain qui confine à une position paradoxale : tout en tirant gloire de leur insuccès dans lequel ils voient une confirmation de leur valeur profonde, ils ont du mal à accepter de ne pas être reconnus à leur juste valeur. Loin de suivre les conseils prudents de Barbey qui l'exhorte à " plaire aux imbéciles de L'Univers » (Barbey d'Aurevilly, 1987, p. 181), le disciple radicalise la violence du maître au lieu de l'atténuer. C'est ainsi qu'il ne cesse de se faire remercier par les journaux, avec lesquels il ne collabore jamais très longtemps.

Barbey se fait en outre son mentor dans le monde des Lettres; il guide Bloy au sein d'un monde qui reste encore inintelligible à ce dernier. Il lui ouvre les portes de L'Univers par l'intermédiaire de Blanc de Saint-Bonnet, là où son père biologique lui avait ouvert les portes d'un cabinet d'architecte à Paris pour travailler comme commis lorsque Bloy était arrivé à la capitale à dix-huit ans. Il le pousse en écrivant la préface louangeuse de son premier essai, autre manière d'asseoir une position magistrale. Faire une préface revient à se porter garant. Façon de légitimer et donc de faire valoir l'auteur de l'ouvrage en question, c'est une position d'autorité au sens étymologique du terme, qui rejoint l'idée d'augere : par sa préface, le maitre augmente la valeur symbolique de la création de son disciple. Il est l'auctor, le fondement de l'autorité, au sens où il prend la responsabilité de ce que son disciple a pu produire.

Davantage qu'un mentor, Bloy trouve en Barbey l'« éducateur de [son] intelligence " (Bloy, 1965, p. 24), un tuteur. Le Connétable lui fait lire les "Prophètes du Passé », il corrige ses premiers articles. Bloy est demandeur. Ainsi lorsqu'il prévoit d'écrire son troisième article pour L'Univers à propos de l'amiral de Coligny, il lui écrit : "Vous me rendriez un grand service en me donnant dans une lettre très prochaine deux ou trois idées sur ce Coligny " (Bollery, 1947, p. 187). Contrairement à son père biologique qui voit dans la carrière littéraire une dangereuse perdition, 
son père de substitution l'encourage. Dispensateur de conseils, il le guide dans l'élaboration de son écriture. Il se voit parfois poussé à corriger son élève à l'écriture jugée d'abord trop monotone. Il lui reproche plus largement, dans sa lettre du 4 juillet 1874 , dêtre « trop sérieux » :

Vous êtes trop un écrivain ecclésiastique. Vous m’avez rappelé Laurentie (des bons jours), Laurentie qui se serait, le matin, lavé la bouche avec du de Maistre.

Lisez Voltaire. Lisez les légers et coupez la queue à vos chiens, - non, vous n'en avez pas, - mais à vos chiennes de phrases. Remuez-vous davantage dans les vôtres. Lisez Voltaire. Il ne faut pas tant lire ses analogues. Il faut lire ses différents. (Barbey d'Aurevilly, 1987, p. 201)

C'est alors que le rapport de bienveillance paternelle devient proche d'un rapport de curatelle qui en est sa corruption. À l'occasion d'un retour sur l'orageuse amitié qu'il a entretenue avec Huysmans, Bloy déclare :

jétais en quête de ma personnalité littéraire et je voulais échapper à la tutelle de d'Aurevilly qui, malgré ses défauts, avait du moins, une générosité d'esprit et une chevalerie de cœur qui manquent profondément à Huysmans. Je le sais aujourd'hui. En croyant m’échapper, je tombai dans une tutelle pire. (Bloy, 2012, p. 518)

Tout lecteur de la correspondance entre Bloy et Barbey ne manque pas de remarquer la tentation qu'a Bloy de déléguer la responsabilité de choix cruciaux à son maître, fort mal à son aise dans ce rôle. Ces différentes diffractions du rapport filial montrent à quel point celui-ci est structurant. Cet imaginaire profus doit beaucoup au contexte social et culturel marqué par une crise communautaire forte qui affecte, entre autres, la cellule familiale ${ }^{12}$. De même que la remise au goût du jour des figures du maître et du disciple dans les années 1890, notamment à travers le genre du roman à thèse, procède en partie d'une réaction à la vogue littéraire décadente de la décennie précédente, de même, c'est sans doute à la faveur d'une telle crise qu'a été rendu possible l'établissement de ce rapport dont le caractère magistral s'épanouit dans un cadre filial.

Un tel imaginaire couronne le déploiement d'une pensée par des images qui, par leur symbolique, renferment l'essentiel. Celles-ci sont radicales, ardentes ; en elles, nulle tiédeur. Leur sphère est celle de l'idéal, catégorie dont on sait combien elle fédère alors les opposants au naturalisme et à tous les tenants de l'observation exacte, jugée fallacieuse, du réel. Barbey disait de Bloy qu’il voyait « énorme » (Bloy, 1875 , p. 5). Une pareille vision ne pouvait que déboucher sur l'emballement ardent

12. Le foisonnement théorique de la sociologie naissante sur ce sujet est révélateur du fait que le lien communautaire, traditionnellement pensé sous un prisme aristotélicien, devient un objet problématique. 
d'un imaginaire au sein duquel l'image pense : "Sous l'image toujours pompeuse, note Barbey dans les marges du manuscrit de La Méduse-Astruc, il y a de la pensée ou du sentiment »(Bloy, 1875, p. 6).

\section{RÉFÉRENCES}

Barbey d’Aurevilly, J. (1987). Correspondance générale. P. Berthier et A. Hirschi (dir.), vol. VII. Paris : les Belles lettres.

Barbey d'Aurevilly, J. (1988). Correspondance générale. P. Berthier et A. Hirschi (dir.), vol. VIII. Paris : les Belles lettres.

Bloy, L. (1875). La Méduse-Astruc. Signé : Léon Bloy. Lettre et annotations de J. Barbey d'Aurevilly. Paris : Bibliothèque nationale de France, département Réserve des livres rares, RES M-Z-406 (2).

Bloy, L. (1964). Le Révélateur du Globe. Dans J. Bollery et J. Petit (éd.), CEuvres de Léon Bloy, vol. I (p. 17-252). Paris : Mercure de France.

Bloy, L. (1965). La Méduse-Astruc. Dans J. Bollery et J. Petit (éd.), CEuvres de Léon Bloy, vol. IV (p. 19-33). Paris : Mercure de France.

Bloy, L. (1967). Sueur de sang. Dans J. Bollery et J. Petit (éd.), CEuvres de Léon Bloy, vol. VI (p. 13-190). Paris: Mercure de France.

Bloy, L. (1999). Journal I. P. Glaudes (éd.). Paris : Robert Laffont.

Bloy, L. (2012). Léon Bloy. Louis Montchal et Henriette L'Huillier. Correspondance (1884-1906). G. Guyot-Rouge (éd.). Paris : Classiques Garnier.

Bollery, J. (1947). Léon Bloy. Origines, jeunesse et formation (1846-1882). Paris : Albin Michel. Bollery, J. (1949). Léon Bloy. Ses débuts littéraires du Chat Noir au Mendiant Ingrat (18821892). Paris : Albin Michel.

Petit, J. (1971). Barbey et ses épigones, Revue des lettres modernes, 6, 7-22.

Racine, J. (1999). Phèdre. G. Forestier (éd). Paris : Gallimard.

Weber, M. (1959). Le savant et le politique. Paris : Plon.

RÉSUMÉ : Père de substitution, mentor, tuteur, objet d'admiration et d'imitation, Barbey d'Aurevilly remplit pour Bloy, depuis leur rencontre, les fonctions d'un maître. Parmi tous ceux qui se revendiqueront d'un héritage de Barbey, Bloy semble en effet avoir été le disciple le plus fervent du Connétable des Lettres. Tout en nous concentrant en particulier sur la manière dont les figures d'autorité qui renvoient à Barbey se construisent chez Bloy, nous nous proposons de voir comment ces deux écrivains, qui sont entièrement du côté du figuratif, donnent une densité particulière à leur rapport à travers le travail de l'image qui prend une place centrale et essentielle dans la construction du lien entre un maître et un disciple. Cette enquête nous amènera en outre à considérer qu'une telle relation ne va ni sans prise de distance ponctuelle ni sans agacement réciproque. Loin de s'effacer devant le maitre, Bloy se construit clairement moins dans l'ombre que dans la lumière d'un maître qui l'éclaire. 
Mots-clés : Bloy, Barbey d’Aurevilly, imaginaire, maître, disciple

\begin{abstract}
"The man to whom I owe the most, after my mother and father": Léon Bloy, Jules Barbey d'Aurevilly's disciple

ABSTRACT: As a substitute father figure, a mentor, a tutor and a writer to be admired and imitated, Barbey d'Aurevilly acted as a master for Bloy from the moment they first met. Indeed, among all the other self-proclaimed heirs of Barbey's legacy, Bloy seems to be the most ardent disciple of the Connétable des Lettres. As we concentrate on the way Bloy constructs multiple authority figures who all somehow relate to Barbey, we propose to study the way these two writers, who both lean exclusively towards the figurative, give a particular depth to their relationship by elaborating their imagery, which plays an essential role in the way they construct the bond between master and disciple. This investigation will, in addition, lead us to consider how such a relationship cannot come about without any occasional differences of opinion or mutual irritation. Far from shying away from the master, Bloy clearly constructs his own authorial persona in the light of the brilliant master, rather than in his shadow.
\end{abstract}

Keywords: Bloy, Barbey d'Aurevilly, imagination, master, disciple 